\title{
Long-Lasting Working Memories of Obstacles Established by Foreleg Stepping in Walking Cats Require Area 5 of the Posterior Parietal Cortex
}

\author{
David A. McVea, ${ }^{1}$ Andrew J. Taylor, ${ }^{2}$ and Keir G. Pearson ${ }^{2}$ \\ ${ }^{1}$ Graduate Program in Neuroscience, University of British Columbia, Vancouver, British Columbia V6T 1Z3, Canada, and ${ }^{2}$ Department of Physiology, \\ University of Alberta, Edmonton, Alberta T6G 2H7, Canada
}

Walking animals rely on working memory to avoid obstacles. One example is the stepping of the hindlegs of quadrupeds over an obstacle. In this case, the obstacle is not visible at the time of hindleg stepping, because of its position between the fore and hindlegs, and working memory must be used to avoid it. We have previously shown that this memory is very precise and surprisingly long-lasting and that it depends on the stepping of the forelegs over the obstacle for its initiation. In this study, we test the hypothesis that area 5 in the posterior parietal cortex of cats is necessary for the maintenance of this long-lasting working memory. We report that small bilateral lesions to area 5 do not affect the amplitude of normal stepping of the hindlegs over obstacles, but they profoundly reduce the long-lasting working memory of obstacles. We propose that inputs to area 5 associated with foreleg stepping initiate long-lasting activity that maintains the memory of obstacle height in another brain region to guide the hindlegs over obstacles.

\section{Introduction}

The study of the visual control of walking has revealed many of the strategies used to detect and avoid obstacles in humans (Patla, 1997; Patla and Greig, 2006; Marigold et al., 2007; Cinelli and Patla, 2008; Marigold and Patla, 2008) and animals (Drew, 1993; Bellozerova and Sirota, 2003; Drew et al., 2008). One consistent theme that has emerged is that visual fixation of obstacles is rarely used to guide ongoing leg movements. Instead, visual information relevant to walking is gathered in advance and then used later to alter leg movements (Patla and Vickers, 1997; Fowler and Sherk, 2003; Wilkinson and Sherk, 2005).

In quadrupeds, there is a particular need for some form of memory to guide the hindlegs, because the hindlegs approach an obstacle after the head and eyes have moved past. Recently, our laboratory has begun to investigate the characteristics of this memory in cats. We have discovered that it is very long-lasting (many minutes) compared with the duration of many working memory tasks (Pasternak and Greenlee, 2005; Fiset and Doré, 2006) and is used to precisely guide the movements of the hindlegs (McVea and Pearson, 2006). Furthermore, this long-lasting working memory of obstacle size and location is only established if the forelegs have already stepped over the obstacle. If a cat is stopped just before stepping over an obstacle, the memory of its size and location decays over seconds (McVea and Pearson, 2007). We have suggested that an efference copy of foreleg motor

Received Feb. 8, 2009; revised June 3, 2009; accepted June 5, 2009.

The research was supported by a grant from the Canadian Institutes of Health Research. We thank Rod Gramlich for technical support.

Correspondence should be addressed to David A. McVea, Brain Research Centre, University of British Columbia, 4N1-2255 Westbrook Mall, Vancouver, BC V6T 1Z3, Canada. E-mail: dmcvea@interchange.ubc.ca.

D0I:10.1523/JNEUROSCI.0746-09.2009

Copyright $\odot 2009$ Society for Neuroscience $\quad$ 0270-6474/09/299396-09\$15.00/0 commands likely combines with visual information about an obstacle's size and location to establish a neural representation of obstacle location relative to the body that persists while the animal is straddling the obstacle.

Although the neural structures that establish the persistent memory of a straddled obstacle are unknown, evidence suggests the posterior parietal cortex (PPC) is involved. First, the posterior parietal cortex is involved in establishing an egocentric representation of objects in the external world relative to the body (Burgess, 2006) and for the online planning control of visually guided movements (Buneo and Andersen, 2006). Second, lesions of the posterior parietal cortex lead to deficits in the accuracy of reaching movements to remembered targets in humans (BattagliaMayer and Caminiti, 2002), and recordings from neurons in area 5 in monkeys have revealed neurons that remain active during the delay period between target presentation and movement onset (Kalaska, 1996). Third, lesions of the posterior parietal cortex in cats impair the accuracy of reaching movement to moving targets (Fabre and Buser, 1981) and stepping movements to avoid obstacles (Lajoie and Drew, 2007). And finally, a preliminary study has reported that some neurons in area 5 of the posterior parietal cortex in walking cats remain active while an animal straddles an obstacle between its fore and hindlegs (Lajoie et al., 2007).

In this investigation, we have tested the hypothesis that area 5 in the posterior parietal cortex is necessary for establishing the persistent memory of an obstacle after the forelegs have stepped over the obstacle. If this hypothesis is correct, then we made the prediction that the persistent, long-lasting working memory of an obstacle would be abolished after lesions of area 5. A preliminary report of our findings has been published previously (Pearson and McVea, 2007). 


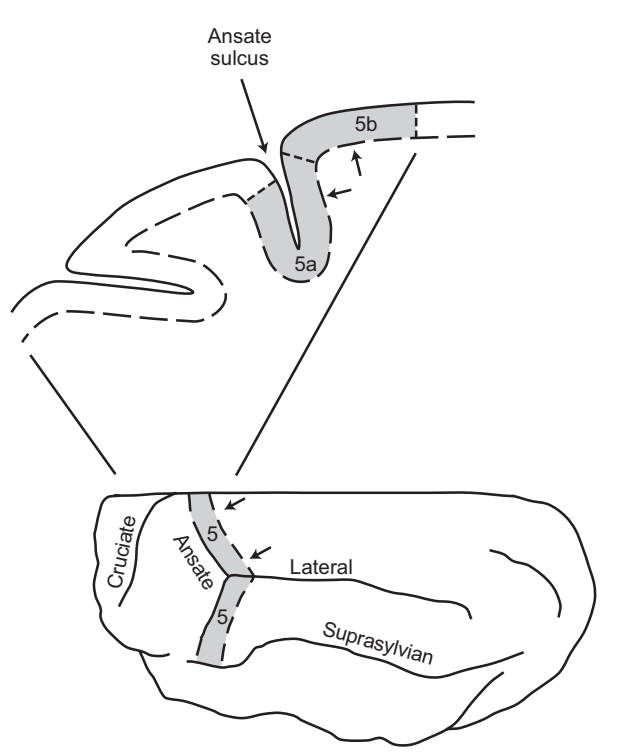

Figure 1. Location of lesions in area 5 of the posterior parietal cortex. Area 5 (shaded) extends from the midline to the suprasylvian gyrus and is located within and caudal to the ansate sulcus. Lesions were made caudal to the ansate sulcus (indicated as the region between the 2 arrows in the top and bottom diagrams) close to the division between area $5 \mathrm{a}$ and area $5 \mathrm{~b}$ in the medial region of area 5 .

\section{Materials and Methods}

All experimental procedures were reviewed and approved by the Health Sciences Animal Welfare Committee at the University of Alberta. Experiments were performed with four adult cats (one male and three females), ranging in weight from 3.5 to $5 \mathrm{~kg}$. The goal of the experiments was to determine the effect of lesioning area 5 in the PPC on an animal's ability to remember the location of an obstacle relative to the body. The location of area 5 in the cat is shown in Figure 1 [for more detailed anatomy, see Ghosh (1997); Andujar and Drew (2007)].

Behavioral task. The behavioral task has been described in previous publications (McVea and Pearson, 2006, 2007). Briefly, each cat was trained to walk along a walkway $(2.5 \mathrm{~m} \times 30 \mathrm{~cm})$ and stop walking when it had stepped over an obstacle (height, $7.2 \mathrm{~cm}$ ) with its forelegs but not with its hindlegs (Fig. $2 \mathrm{~A}$ ). While the cat was straddling the obstacle, the obstacle was removed by lowering it into a slot in the walkway. The animal then stood straddling the original location of the obstacle for periods ranging from $\sim 1 \mathrm{~s}$ to $2 \mathrm{~min}$. This posture was maintained by allowing the cat to eat soft food from a dish placed in front of it. Walking was subsequently initiated by moving the feeding dish forward. In normal animals, both hindlegs are usually lifted higher so that the paws pass over the original location of the top of the obstacle (McVea and Pearson, 2006). A movie of a representative trial is available at www.jneurosci.org as supplemental material. In all cases, food was provided to motivate the animal to walk forward, regardless of the animal's behavior, and no rewards were given for any particular outcome, i.e., the stepping of the hindlegs over the obstacle was not specifically rewarded. The reason for removing the obstacle was to prevent the paws contacting the obstacle and thus eliminate the possibility that the animal could learn a tactile withdrawal response in the context of the task. Trials in which the cat sat down, stepped backwards, crouched, or any other behavior that could give it a clue that indicated that the obstacle had been removed were excluded from analysis, regardless of subsequent behaviors.

Two types of control trials were also carried out. The first was to allow each animal to step normally over the obstacle with all four legs without pausing. In this case, the obstacle was straddled between the forelegs and hindlegs for periods in the range of $0.5 \mathrm{~s}$ with the exact value depending on normal variations of walking speed. The second was to lower the obstacle before the trial began and to stop the animal after its forelegs had stepped over the lowered obstacle. In this situation, the top of the obstacle was flush with the surface of the walkway and clearly visible as the animal approached the feeding dish.
A
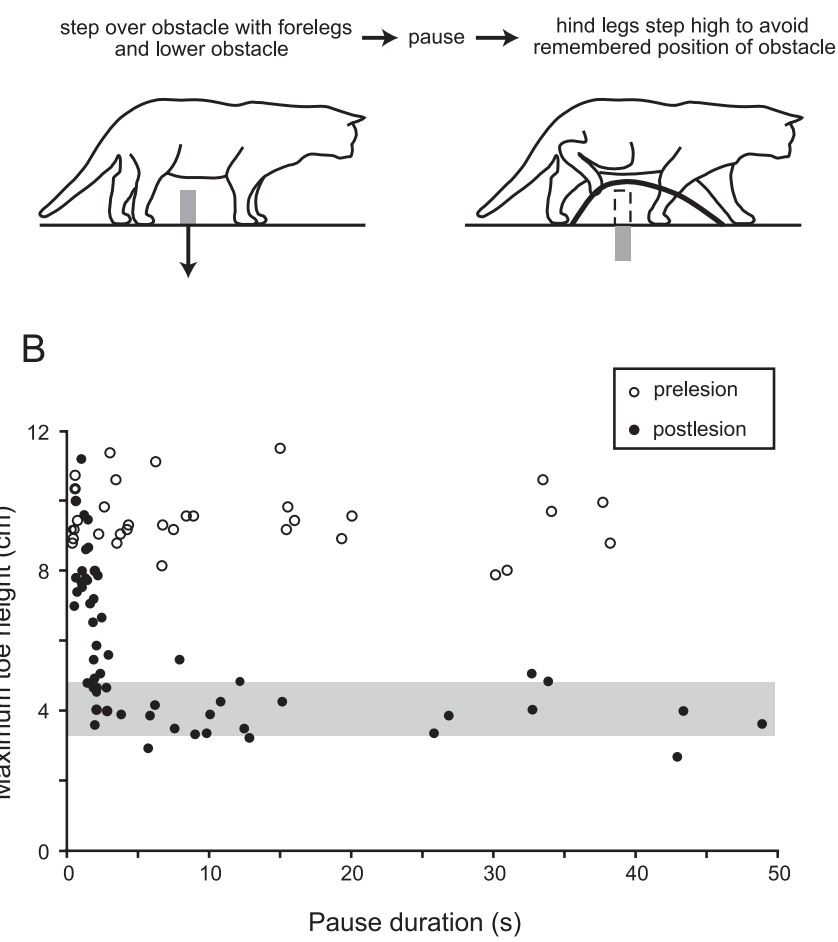

Figure 2. Bilateral lesions of area 5 cause the loss of a long-lasting working memory of an obstacle. $A$, Task for measuring memory of an obstacle. An animal walks forward, steps over an obstacle with the forelegs, and pauses. While paused, the obstacle is removed, and some time later the animal resumes walking. Memory of the obstacle is indicated by an elevated trajectory of the paw (thick line) over the previous location of the obstacle. See supplemental video, available at www.jneurosci.org as supplemental material. $\boldsymbol{B}$, Example of memory loss in cat 2 . Scatter plot showing the maximum toe height versus pause duration before (open circles) and $2 \mathrm{~d}$ after (filled circles) bilateral lesions of area 5 . The shaded area shows the range of toe heights (mean \pm SDs) when the obstacle was not present at any time. Before the lesions, the memory of obstacle height was maintained for tens of seconds. After the lesions, there was a complete loss of memory for pause durations greater than $\sim 5 \mathrm{~s}$. Note that after the lesions there was little or no loss of memory for very short pause durations.

The type of trial (control or experimental) and the pause durations were not explicitly randomized, because we have never observed a decline in memory duration with repeated trials at the same pause duration. Nevertheless, pause durations were chosen in an irregular manner with the goal of observing the performance over a range of delays on each day. The exact duration of the delay for each trial was later determined from the video recordings. As a result, the features of each trial were unpredictable to the animal.

Surgical procedures. In three animals, lesions were made in area 5 in both hemispheres during the same surgical procedure. In the fourth cat, area 5 in only the right hemisphere was lesioned. In the rostral-caudal direction, area 5 begins in the rostral banks of the ansate sulcus and extends caudally to the ansate gyrus, whereas in the medial-lateral direction, area 5 begins in at the midline and extends beyond the lateral sulcus to the suprasylvian sulcus. Anatomical studies on the projection of neurons to and from area 5 has led to the division of this area into two subregions: area $5 \mathrm{a}$ projecting to the caudal motor cortex and receiving primarily cutaneous sensory inputs and area $5 \mathrm{~b}$ projecting to the rostral motor cortex and receiving primarily proprioceptive and visual sensory inputs (Ghosh, 1997; Andujar and Drew, 2007). Furthermore, there is evidence for a crude topographical mapping of area 5 onto the motor cortex such that lateral and medial regions tend to project to foreleg and hindleg regions of the motor cortex, respectively. Because the focus of the present study was establishing whether area 5 was involved in establishing the long-lasting working memory for the guidance of movements of 
the hindlegs over obstacles, we chose to confine our lesions to the medial region of area 5. Moreover, we also limited the lesions to the ansate gyrus and part of the caudal bank of the ansate sulcus, because preliminary studies have found single neurons in this region that increase their activity when an obstacle passes under the walking animal (Drew et al., 2008) and when an animal is standing straddling an obstacle between the forelegs and hindlegs (Lajoie et al., 2007).

Cats were prepared for surgery by pretreatment with subcutaneous injections of acepromazine $(0.1 \mathrm{mg} / \mathrm{kg})$, glycopyrrolate $(0.01 \mathrm{mg} / \mathrm{kg})$, and hydromorphone $(0.02 \mathrm{mg} / \mathrm{kg})$, and then anesthetized with isoflurane (2-3\% with oxygen). Anesthesia was maintained throughout the surgery by delivering the anesthetic via an intubating tube inserted into the trachea. Saline was given continuously via an intravenous line on a foreleg at a rate of $8 \mathrm{ml} / \mathrm{kg} / \mathrm{hr}$, and a dose of $30 \mathrm{mg} / \mathrm{kg}$ solumedrol was administered intravenously either before or at the end of the surgical procedure to help reduce cerebral edema. Heart and respiration rates were monitored throughout. Four hours after the surgery, each animal received a subcutaneous injection of an analgesic (hydromorphone, $0.02 \mathrm{mg} /$ $\mathrm{kg}$ ). An antibiotic (Clavomox) was given orally twice daily during the week after the surgery.

When anesthetized, the animal's head was placed in a stereotaxic frame, and small areas $(\sim 1 \times 1 \mathrm{~cm})$ of the skull were removed bilaterally (cats 1 to 3 ) or unilaterally (cat 4 ) to expose the posterior parietal region. The dura was opened and folded back to expose the ansate sulcus and the adjacent gyri. Large blood vessels running over the medial surface of the caudal gyrus were cauterized, and the anterior margin of this gyrus was aspirated. An attempt was made to confine the lesions medial to the lateral sulcus, since this region gives rise to neuronal projections to the hindleg region of the motor cortex (Andujar and Drew, 2007). The arrows in Figure 1 show the approximate extent of the lesions. After the aspiration(s), the dura was folded back over the exposed cortex and covered with Gelfoam. A thin layer of dental acrylic was applied to replace the removed portion of the skull.

Anatomy of the lesions. After all trials were completed, the cats receiving the simultaneous bilateral lesions were anesthetized with pentobarbital sodium $(40 \mathrm{mg} / \mathrm{kg})$ and then perfused per cardia with formaldehyde to fix the brain. The brain was removed and photographed to determine the surface extent of the lesions. Blocks of the brain containing the lesioned regions ( $\sim 1$ cubic centimeter in volume) were embedded in agar and sectioned perpendicular the ansate sulcus $(200 \mu \mathrm{m}$ sections in cat 1 and $150 \mu \mathrm{m}$ sections in cats $2-4)$. The sections from cat 1 were dehydrated and stained with cresyl violet to enhance contrast, but this was found not be necessary for visualizing the extent of the lesions, so it was not done for sections from all other cats. Photographs of brain sections were enlarged, and the area of the lesion was determined using a planimeter. This allowed us to calculate the volume of the cortex removed.

Data acquisition and analysis. Trials were recorded on video (JVC Color Video Camera) at a rate of $60 \mathrm{frames} / \mathrm{s}$, which were then digitized using a Peak Motus (Vicon Motion Systems) motion analysis system. To track the movement of the paws, reflective markers $(0.5 \mathrm{~cm}$ diameter) were placed on the first and fourth digits of the paws of the hindlimbs, and these markers were illuminated with a bright light placed directly in front of the video camera. The Peak Motus system was used to determine the pause duration as the cat straddled the obstacle, the toe heights during stepping of the hindlegs, and the order in which the cat stepped over the obstacle, i.e., which legs were leading or trailing.

To quantify the retention of a memory for the height of the obstacle, we first measured the maximum height of the toes of the two hindlegs, regardless of where this point occurred relative to the obstacle. This allowed us to specifically examine the memory of obstacle height separate from any modification of kinematics that might have occurred (see Results). Deficits in memory that occurred after lesions of area 5 were then quantified by comparing the maximum toe heights after the lesions to the maximum toe heights before the lesions. This was expressed as a percentage using the following formula:

Percentage memory deficit

$$
\begin{aligned}
& \text { mean maximum toe height prelesion } \\
& =100 \times \frac{- \text { mean maximum toe height postlesion }}{\text { mean maximum toe height prelesion }} . \\
& \text { - mean maximum toe height without obstacle }
\end{aligned}
$$

This formula yields a value of zero if the lesion had no effect on toe height and a value of 100 if the lesion reduced the step height to control values measured when the obstacle was never present. Statistical analysis 
Table 1. Lesion volumes and memory deficits

\begin{tabular}{|c|c|c|c|c|c|c|}
\hline & Cat 1 & & Cat 2 & & Cat 3 & \\
\hline Location of lesion & Right cortex & Left cortex & Right cortex & Left cortex & Right cortex & Left cortex \\
\hline Lesion volume $\left(\mathrm{mm}^{3}\right)$ & 4.8 & 2.8 & 10.8 & 14.6 & 4.8 & 4.7 \\
\hline Memory deficits & Left leg & Right leg & Left leg & Right leg & Left leg & Right leg \\
\hline$\%$ immediate memory deficit & 60 & 40 & 100 & 100 & 81 & 86 \\
\hline$\%$ memory deficit weeks ( $n$ ) after lesion & $7(1)$ & $0(1)$ & $44(2-3)$ & $75(2-3)$ & $58(2-3)$ & $63(2-3)$ \\
\hline
\end{tabular}

\section{Cat 2}

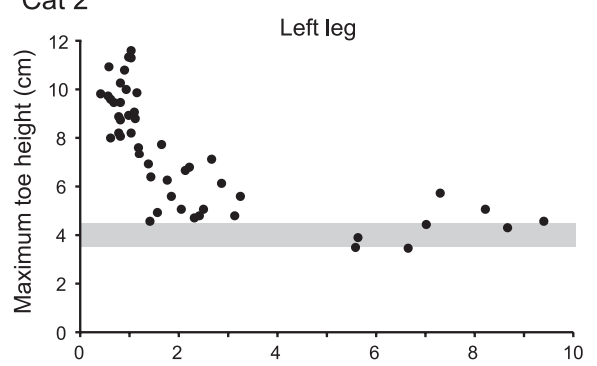

Cat 3
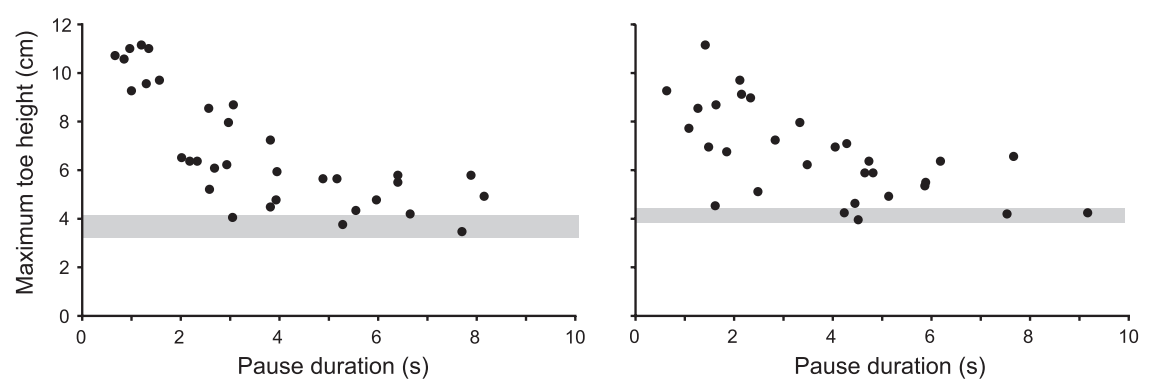

Figure 4. Rapid loss of memory of obstacle height after bilateral lesions in area 5. Scatter plots of maximum toe height versus pause duration in the left and right legs of cat 2 (top) and cat 3 (bottom) after bilateral lesions in area 5 . Note the rapid decline in maximum toe height as pause duration increased over the range of 0.5 to $3 \mathrm{~s}$ in cat 1 and 0.5 to $5 \mathrm{~s}$ in cat 2 .

was performed with Microsoft Excel. The comparison between the means of the different sets of trials was done using the two-tailed Student $t$ test.

\section{Results}

\section{Bilateral lesions of area 5 in posterior parietal cortex}

Bilateral lesions of area 5 were made in a single operative procedure in three adult cats. All animals recovered rapidly from the surgery. On the day after the surgery, all animals displayed their characteristic social behavior and ate normally. No noticeable deficits in stepping and locomotor behavior were observed as the animals walked around the laboratory, stepped over obstacles, and jumped onto chairs and tables. All animals were cooperative when performing the memory task (Fig. 2A).

As predicted, the effect of the bilateral lesions to area 5 was to produce a severe deficit in the animal's ability to remember the height of the obstacle over which the forelegs had stepped. This is illustrated for one animal (cat 2) in the scatter plot in Figure 2 B. This plot shows the maximum toe height of one hindleg as it stepped over the previous location of the obstacle versus the period the animal was paused straddling the obstacle before the lesions (open circles) and on the second day after the lesions (filled circles). Before the lesions, there was little or no reduction in maximum toe height for all pause durations (up to $40 \mathrm{~s}$ ), but after the lesions, there was a complete loss of the long-lasting working memory for pause durations more than $\sim 5 \mathrm{~s}$ [the shaded bar indicates the range of hindleg step heights (mean \pm $\mathrm{SD}$ ) in control trials when there was no obstacle]. Two videos of representative trials (one with short delay, one with long) are shown in the supplemental material, available at www. jneurosci.org. The deficit in long-lasting working memory occurred in both hindlegs in all three animals, as shown in the bar plots in Figure 3. These plots show the mean maximum step height of the left and right legs before the lesions (gray bars) and after lesions (black bars), averaged over two ranges of times from the initiation of stepping of a foreleg over the obstacle to the time a hindleg began to step over the obstacle: $0.5-1.5 \mathrm{~s}$ and $10-40 \mathrm{~s}$. The lower interval range of $0.5-1.5 \mathrm{~s}$ included trials in which the animal was not paused. In all lesioned animals, the maximum step height of both hindlegs was significantly reduced for pause durations in the range of 10-40 s. However, the magnitude of this reduction varied from animal to animal, and in cat 1 , it varied between the two hindlegs. These variations were related to the size and extent of the lesions.

The largest memory deficit was observed in cat 2 . In this animal, the maximum toe height of the hindlegs when stepping over the previous location of the obstacle was not significantly different from controls without the obstacle for pause durations greater than $\sim 5$ s, i.e., deficits of $100 \%$. Of the three animals receiving simultaneous bilateral lesions, this animal had the largest lesions (see later section of details of the anatomy of the lesions). On the other hand, in cat 1, the lesions were the smallest, and in this animal, the memory deficit was the least, being $60 \%$ for the left leg and $40 \%$ for the right leg. The difference in the memory deficit in the two legs of cat 1 was also correlated with a difference in the lesion sizes in the two hemispheres, with the larger deficit in the left leg being associated with a larger lesion in the contralateral right hemisphere. Finally, in cat 3 , the lesion sizes in each hemisphere were approximately equal and intermediate between the lesion sizes in cats 1 and 2 . Correspondingly, the memory deficits in the two hindlegs were similar ( $81 \%$ in left and $86 \%$ in right) and between the deficits observed in cats 1 and 2. Table 1 lists the volume of each lesion and the magnitude of the memory deficit in the associated (contralateral) hindleg in the days immediately after the lesion. The $R^{2}$ value of linear correlation coefficient between these two parameters was 0.61 .

A striking and consistent feature of the stepping behavior of the hindlegs after the lesions was that no memory deficit for obstacle height occurred when the animals walked over the obstacle without pausing or when the pause duration was less than $\sim 1.5 \mathrm{~s}$. In all three animals, the average maximum top height of the hind toes when stepping over the obstacle on short-duration trials was not significantly different in prelesioned and postle- 
sioned animals (Fig. 3). Thus, the memory loss occurring with longer delays (range, 10-40 s) was not attributable to a deficit in the ability to lift the paws, and it explains why deficits were not obvious from general observations of animals walking in the cluttered environment of the laboratory. Another consistent feature was that the memory loss in the lesioned animals occurred quickly after the forelegs had stepped over the obstacle. In two of the animals (cats 2 and 3), we examined the time course of this progressive loss of memory by recording many trials over a range of pause durations from 0 to $10 \mathrm{~s}$ (Fig. 4). In both animals, it was clear that the memory loss increased progressively over a few seconds from the time the forelegs stepping over the obstacle and was complete for both hindlegs within $\sim 3$ s for cat 2 (Fig. $4 A$ ) and within $\sim 5 \mathrm{~s}$ for cat 3 (Fig. $4 B$ ). Insufficient trials over the range of pauses from 0 to $10 \mathrm{~s}$ were recorded in cat 1 to establish this time course of memory loss, but it was clear that the memory loss in this animal was fully established within $10 \mathrm{~s}$.

A recent study by Lajoie and Drew (2007) has examined in detail the coordination and movement accuracy of the hindlegs during stepping over obstacles followed lesions to area 5. Although we did not examine these parameters in detail, we did observe that the lesions produced an increase in the variability of the toe trajectories during control trials when the obstacle was present. In two of the cats (cats 2 and 3), the range of the distance from the point of maximum toe height to the obstacle increased in both the leading and trailing hindlegs after the lesions. This dysmetria occasionally caused a toe of one of the hindlegs to touch the obstacle [also observed by Lajoie and Drew (2007)]. Trials in which the toe touched the obstacle were excluded from our analysis of memory deficit.

\section{Memory recovery after bilateral lesions of area 5}

In all three animals, the memory loss after bilateral lesion of area 5 decreased over a period of 1 to 3 weeks. In cat 1 , this recovery was the fastest and returned close to normal, within a week. This cat had the smallest deficits immediately after the lesions, and it also had the smallest lesions. Table 1 lists the magnitudes of the memory deficits in each hindleg at the time recovery was assessed (at 1 week in cat 1 and between 2 and 3 weeks in cats 2 and 3 ) and the volumes of the lesions in contralateral cortex. The other two animals showed significant but modest improvement in memory at 2 to 3 weeks after lesion, but neither returned to normal by 3 weeks after lesion. In cat 2, the memory deficits for the left and right legs decreased from 100 to $44 \%$ and from 100 to $75 \%$, respectively, and in cat 3 , the memory deficits for the left and right legs decreased from 81 to $58 \%$ and from 87 to $63 \%$, respectively. The asymmetry in the partial recovery of the left and right legs in cat 2 was of interest, because this was associated with an asymmetry in the size of the lesions in the two hemispheres (see below for details). The bigger lesion in the left hemisphere was associated with a smaller recovery in the contralateral right hindleg.

\section{Unilateral lesions of area 5 in posterior parietal cortex}

Two observations from the animals receiving bilateral lesions indicated that the persistence of the memory of an obstacle for controlling a hindleg was primarily dependent on the integrity of the contralateral area 5: in cat 1, the larger deficit in the left hindleg was associated with a larger lesion in the right cortex (Fig. 2 ), whereas in cat 2 , the slower recovery of memory retention in the right leg was associated with a larger lesion in the left cortex. To examine the laterality of the memory system, we performed a unilateral lesion in one animal. Immediately after the lesion (days 1 to 3 ), there was a clear asymmetry in the memory deficit in the
A
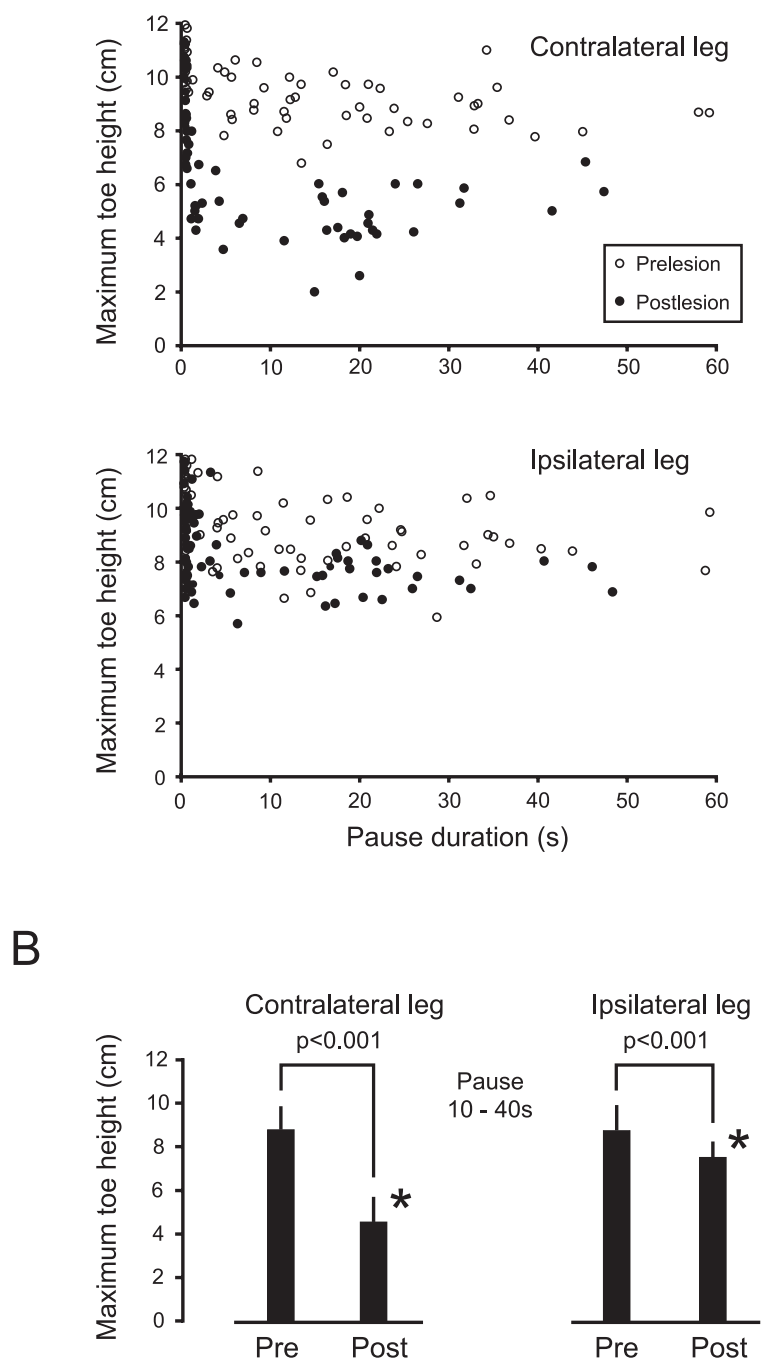

Figure 5. Unilateral lesion of area 5 causes asymmetric loss of memory in the two hindlegs. $\boldsymbol{A}$, Scatter plots showing the maximum toe high versus pause duration in the contralateral (left leg) and ipsilateral (right leg) before (open circles) and after (filled circles) a lesion of area 5 in the right hemisphere. Note the obvious difference in maximum toe heights before and after the lesion in the contralateral leg (top scatter plot) and the relatively small effect of the lesion on toe heights in the ipsilateral leg (bottom scatter plot). $\boldsymbol{B}$, Bar plots showing means of maximum toe heights in the legs contralateral and ipsilateral to the lesion before (pre) and after (post) the lesion when the animal was paused for periods ranging from 10 to $40 \mathrm{~s}$. Note that the lesion caused a significant $\left({ }^{*}\right)$ memory loss in both legs, with the loss in the contralateral leg being larger. Error bars are SDs, and the number of trials for each condition ranged from 22 to 28 .

two hindlegs (Fig. 5), with the deficit being much larger in the hindleg contralateral to the lesion. The long-lasting working memory loss in the contralateral hindleg was $67 \%$, whereas in the ipsilateral leg, it was $21 \%$. The latter was significantly greater than zero, thus demonstrating that, in this animal at least, the persistence of memory of the obstacle in one hindleg was not strictly dependent on the neuronal systems in contralateral area 5.

Two general features of the memory loss in the contralateral (left) leg were similar to those in the animals receiving the bilateral lesions. First, when the animal was not paused (control trials with obstacle), the maximum toe height was similar to that in the same leg before the lesions, and second, the loss of memory occurred rapidly over a period of a few seconds (Fig. 5A, top).

In the animal receiving the unilateral lesion, there was also a 
Cat 1

Cat 2
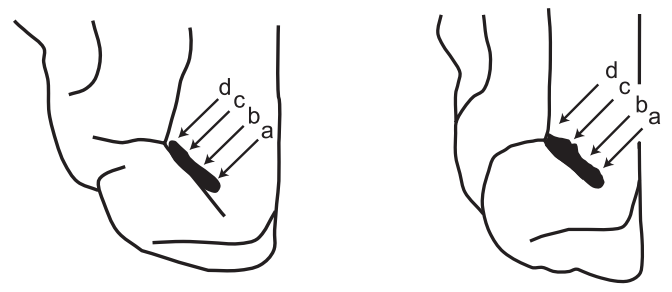

$0.5 \mathrm{~cm}$

a

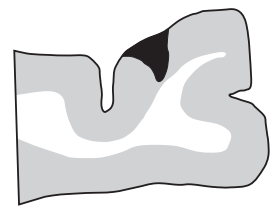

b

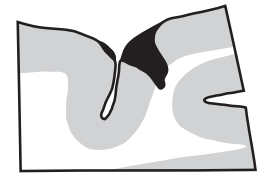

C

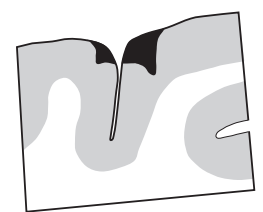

d

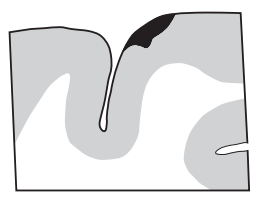

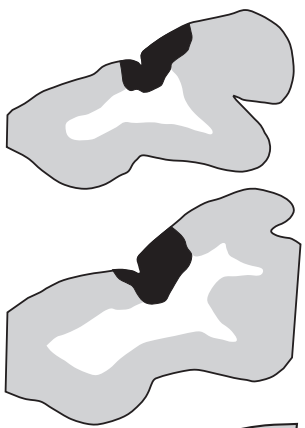
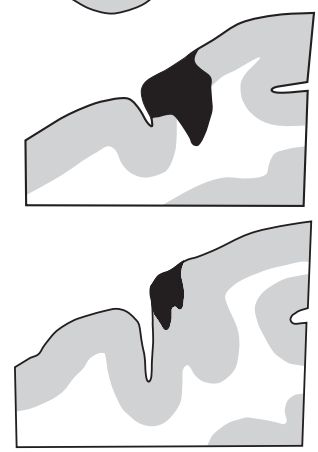

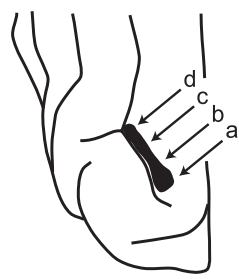

Cat 3
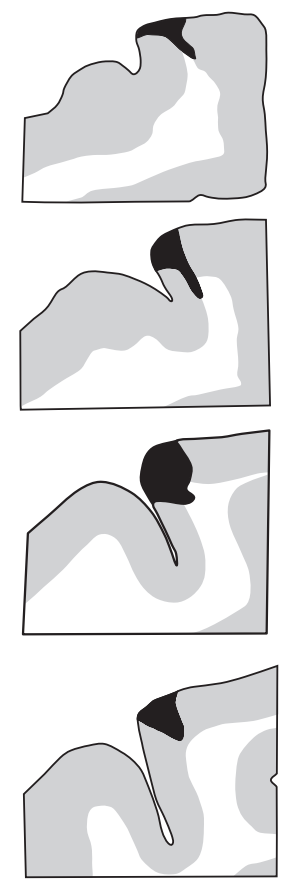

Figure 6. Anatomy of lesions in area 5 of the right hemisphere of the three cats receiving simultaneous bilateral lesions (cats 1-3). The extent of the lesions in the posterior parietal cortex is shown at top of each column, and four cross-sections at different points in the lesion are shown in the columns. Rostral is to the left of each section. The locations of the cross-sections in rows $a-d$ are indicated by the arrows in the top panels.

large, significant recovery over a $2-3$ week period of the longlasting working memory in hindleg contralateral to the lesion and a smaller but just insignificant $(p=0.066)$ recovery in the ipsilateral hindleg. However, the average maximum toe heights in both hindlegs remained significantly lower than prelesion values. The deficits 2 to 3 weeks after the lesion were 13 and 14\% for the contralateral and ipsilateral legs, respectively, compared with 67 and $21 \%$ over the $2 \mathrm{~d}$ immediately after the lesion.

\section{Anatomy of lesions}

Serial sections perpendicular to the ansate sulcus revealed that in all but one case the lesions were primarily confined to the gyrus caudal to the ansate sulcus, did not extend to the fundus of the sulcus, and confined to the medial part of area 5 between the lateral sulcus and the midline (Figs. 6,7 ). The one exception was the lesion to the left hemisphere in cat 2 (Fig. 7, middle). In this animal, the ansate sulcus was relative shallow, and on the left side, both the rostal and caudal banks of the sulcus were removed over a portion of the lesion. The volumes of tissue removed in the left and right hemispheres, respectively, were cat $1,2.8$ and $4.8 \mathrm{~mm}^{3}$; $\overline{2 \mathrm{~mm}}$

cat $2,14.6$ and $10.8 \mathrm{~mm}^{3}$; and cat $3,4.7$ and $4.8 \mathrm{~mm}^{3}$. In all cases, the lesions extended to varying extents into the underlying white matter. The amount of white matter removed was the smallest in cat $1,0.09$ $\mathrm{mm}^{3}$ on the left and $0.27 \mathrm{~mm}^{3}$ on the right, intermediate in cat $3,0.31 \mathrm{~mm}^{3}$ on left and $0.13 \mathrm{~mm}^{3}$ on the right, and largest in cat 3, $3.02 \mathrm{~mm}^{3}$ on the left and 2.05 $\mathrm{mm}^{3}$ on the right. The relatively small amount of white matter involvement in cats 1 and 3 indicates that damage to white matter was not a major factor in producing the memory deficits in these animals.

Our lesions did not destroy all of area 5 medial to the lateral sulcus, as can be seen in the spared gray matter in the crosssection in Figures 6 and 7. More specifically, with the exception of the left hemisphere in cat 2, almost all of the gray matter in the rostral bank and fundus of the ansate sulcus was spared. There was also considerable sparing of gray matter in the caudal bank of the ansate sulcus in both hemispheres in cats 1 and 3. Based on the anatomical study by Andujar and Drew (2007), it is quite clear that the lesions were located close to the junction of area $5 \mathrm{a}$ and $5 \mathrm{~b}$ and extended more into area $5 \mathrm{~b}$ than into area $5 \mathrm{a}$ [compare section in Figs. 6 and 7 with Andujar and Drew (2007), their Fig. 5].

The anatomy of the lesion in the right hemisphere in cat 4 (the animal used to examine the effect of a unilateral lesion) was similar $\left(4.4 \mathrm{~mm}^{3}\right)$ to the lesions in cat 3. Correspondingly, similar to cat 3 , the long-lasting working memory deficit in the contralateral leg (left) in cat 4 was incomplete (67\%) (Fig. 5A, top) and in between the long-lasting working memory deficits observed in cats 1 and 2 .

\section{Discussion}

When walking cats are paused after their forelegs have stepped over an obstacle, information about the location and height of the obstacle is retained in memory to guide movements of the hindlegs over the obstacle when the animals resume walking (McVea and Pearson, 2006, 2007). A characteristic feature of this memory is its long duration, lasting many minutes without loss. In this study, we tested the hypothesis that area 5 of the posterior parietal cortex is involved in this memory system. This hypothesis was confirmed by the finding that small bilateral lesions of area 5 produced marked deficits in an animal's ability to retain the longlasting working memory of a straddled object. These deficits were present in all animals and were apparent during the first testing after surgery, strongly suggesting they reflect a loss of a functional role of the lesioned area and not adaptive changes in other parts of the brain. The memory deficit was quantified by comparing the maximum height each hindpaw was lifted before and after the lesions when stepping above the location of an obstacle over which the forelegs had stepped. The main finding of this investigation was a rapid and significant decline over time in the 
maximum toe heights of the stepping hindlegs in animals with bilateral lesions of area 5 (Figs. 2-4; supplemental videos, available at www.jneurosci.org as supplemental material).

\section{Role of the parietal cortex in memory- guided hindleg movements}

Recent studies have reported a role of the parietal cortex of the cat during complex walking and reaching tasks (Fabre and Buser, 1981; Beloozerova and Sirota, 2003; Lajoie and Drew, 2007), so it is reasonable to consider whether the loss of memory after a lesion in area 5 is attributable to a disruption of neuronal systems involved in the basic motor control of stepping over obstacles in the absence of delays. We believe this to be unlikely. There were no obvious deficits of normal locomotion after the lesions, and the height of steps without delays over obstacles was not significantly changed after the lesions. This is consistent with another study involving lesions of the parietal cortex in the cat [see Lajoie and Drew (2007), their Figs. 3 and 11]. We consider this strong evidence that sensory systems involved in perceiving obstacles during walking, and motor systems involved in generating and sending flexion commands to muscles, remained intact.

Although the heights of steps over obstacles without delays were not significantly changed after lesions to area 5, step heights after long pauses over obstacles were strikingly reduced (Figs. 2, 3; supplemental videos, available at www.jneurosci. org as supplemental material). Thus, it appears that the lesions specifically impacted a system involved in representing memories of obstacles used to guide future, intended movements. Many previous studies in nonhuman primates have suggested such a role for neuronal systems in the parietal cortex. For example, neurons in the lateral intraparietal (LIP) region of area 7 show spatially tuned activity throughout delay periods before saccades to remembered targets (Gnadt and Andersen, 1988; Barash et al., 1991; Zhang and Barash, 2004) and temporary inactivation of LIP results in decreases accuracy of saccades that is significantly greater for memory-guided saccades than for visually guided saccades ( $\mathrm{Li}$ et al., 1999). In addition, some neurons in area $7 \mathrm{a}$ are active through a delay period during which monkeys remember a particular spatial location, even if no subsequent eye movement is required to this location (Constantinidis and Steinmetz, 1996). Similarly, during reaching movements, neurons in area 5 are active during delay periods before arm movements are initiated (Kalaska and Crammond, 1995), their activity reflects the direction of the upcoming movement (Crammond and Kalaska, 1989), and they are activated when movements are withheld (Kalaska and Crammond, 1995). A noteworthy difference between previous studies on nonhuman primates and our current observations on stepping in cats is the relatively short durations of the memory tasks in the nonhuman
Cat 2

Cat 3
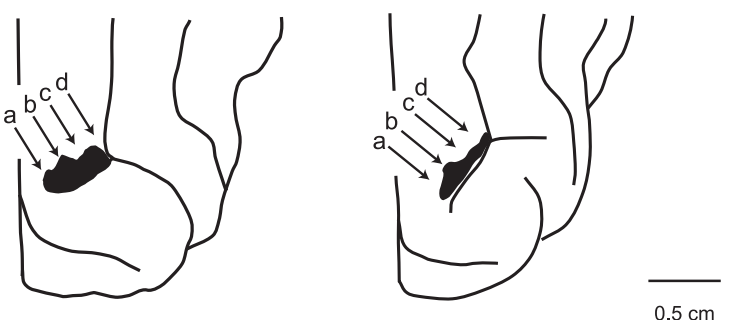

$0.5 \mathrm{~cm}$
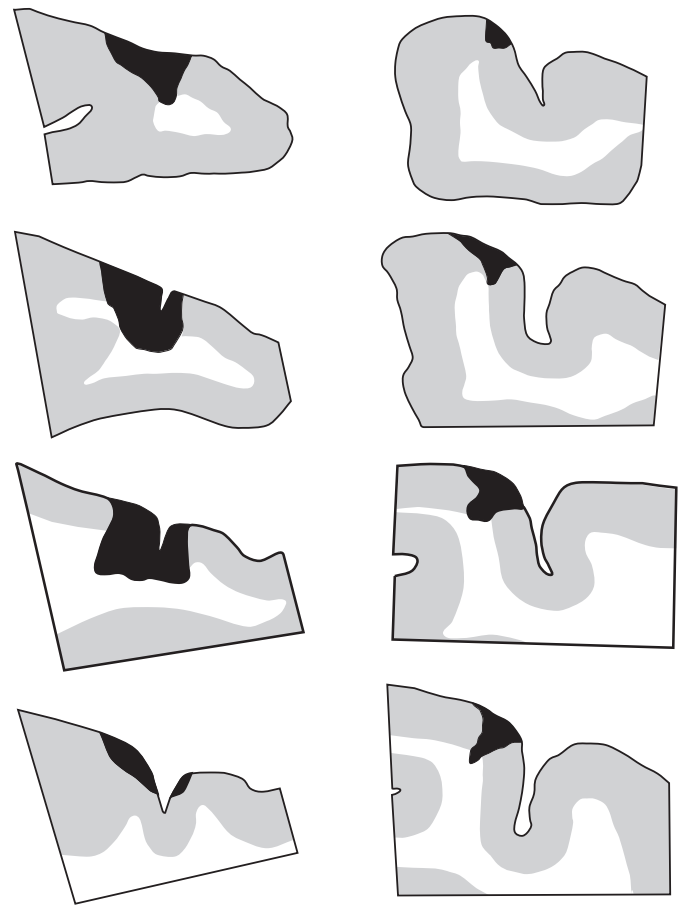

$2 \mathrm{~mm}$

Figure 7. Anatomy of lesions in area 5 of the left hemisphere of the three cats receiving simultaneous bilateral lesions (cats $1-3)$. The extent of the lesions in the posterior parietal cortex is shown at top of each column, and four cross-sections at different points in the lesion are shown in the columns. Rostral is to the right of each section. The locations of the cross-sections in rows $a-d$ are indicated by the arrows in the top panels.

primates and, consequently, the absence of evidence that the posterior parietal cortex in nonhuman primates is involved in establishing long-lasting working memories (lasting for minutes) to guide eye and hand movements to remembered targets.

\section{Foreleg stepping is required to establish long-lasting working memory}

Although our current study suggests that spatial memories of objects encountered while walking might be stored in area 5 for long delays but elsewhere for shorter delays, we must also consider that the duration of memories of obstacles depends crucially on whether or not the forelegs have stepped over the obstacle. Straddled obstacles are remembered for very long durations (up to at least $10 \mathrm{~min}$ ) (McVea and Pearson, 2006), whereas obstacles that are not straddled are remembered for much shorter durations (McVea and Pearson, 2007). In fact, the time course of the memory loss of an obstacle perceived only visually (i.e., in the absence of the forelegs stepping over the obstacle) in intact cats is very similar to the time course of loss we observed in our animals 

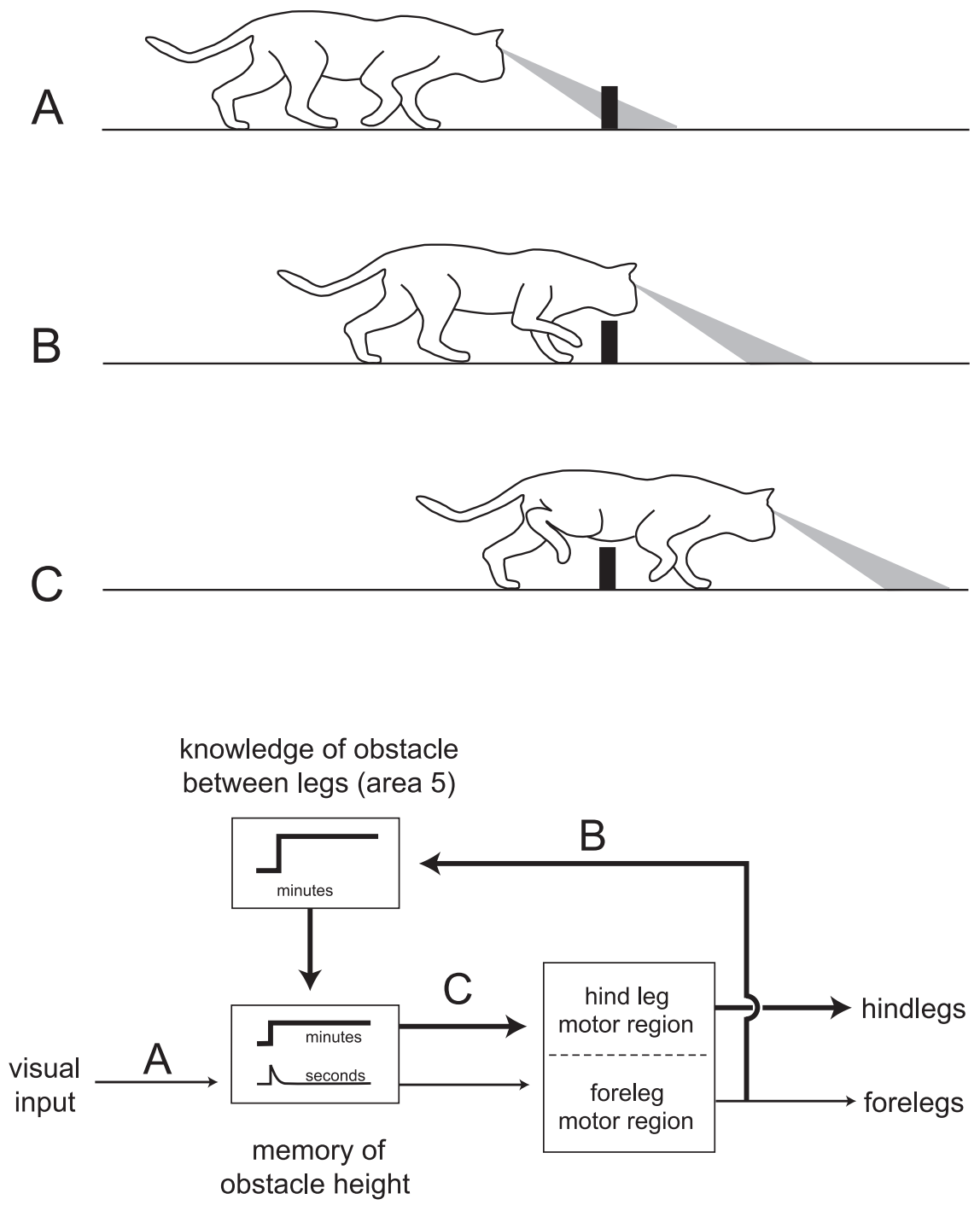

Figure 8. Conceptual model for the role of area 5 in the long-lasting working memories of straddled obstacles. Events in panels A-C (top) correspond to the activation of pathways labeled A-C in the block diagram (bottom). Thin traces represent pathways guiding foreleg movement, and thick traces represent pathways guiding hindleg movements involving long-lasting working memory. $\boldsymbol{A}$, Visual information about the height of an obstacle is stored in memory for a short period of time in an area outside area 5 , indicated by the inset labeled "seconds" in the bottom box. $\boldsymbol{B}$, An efference copy signal related to motor commands producing stepping in the forelegs initiates activity in neurons in area 5 that is maintained for the duration that animal straddles the obstacle, indicated by the inset labeled "minutes" in the top box. Prolonged activity in area 5 then leads to the maintenance of the memory of obstacle height, indicated by the inset labeled "minutes" in the bottom box. C, Motor commands for producing swing movements of the hindlegs are regulated by the memory of obstacle height to lift the hindleg over the obstacle.

with bilateral lesions when they straddled the obstacle [compare Figs. 2, 4, and 5 with McVea and Pearson (2007), their Fig. 1]. Thus, the long-lasting working memory of an obstacle depends on both the presence of intact area 5 and the movement of the forelegs over the obstacle. Information about the form of the obstacle (height and width) must be retained for as long as an animal is straddling an obstacle.

\section{Conceptual model for memory-guided hindleg movements}

A conceptual model that explains these key features of the longlasting working memory is shown in Figure 8. First, we propose that a short-lasting memory of an obstacle is generated in a region outside area 5 as the obstacle passes through the field of vision as the animal approaches the obstacle. This is based on our finding that the memory of obstacle height in lesioned animals is not influenced for short pauses. This memory is used to generate appropriate activity in the foreleg region of the motor cortex to lift the forelegs over the obstacle and in the hindleg region of the motor cortex to lift the hindlegs over the obstacle if the animal does not pause (this portion of our model has been well studied) (Drew et al., 2008). We propose that area 5 receives a signal related to the enhanced flexion of the forelegs and that this signal leads to maintained activity in neurons in area 5 while the animal is straddling the obstacle. A preliminary report has described persistent activity of neurons in area 5 when animals straddle an obstacle (Lajoie et al., 2007). Next, we propose that this maintained activity in area 5 neurons causes the initial short-lasting visual memory of the obstacle outside area 5 to persist until the hindlegs step over the obstacle. The elevated activity of neurons in area 5 may be part of an egocentric representation of the location of the obstacle under the body. This would be consistent with the conclusion from numerous studies on other animals that systems within the posterior parietal cortex play a major role in the egocentric representation of objects relative to the body (Burgess, 2006).

What could be the nature of the suggested signal related to the movement of the forelegs (Fig. 8, pathway B)? Area 5 receives inputs from many regions that include the somatosensory cortex (Avendaño et al., 1988), the motor cortex (Ghosh, 1997), and the cerebellum (Kakei and Shinoda, 1990). Thus, the putative input could be sensory signals generated by the altered trajectory as the forelegs step over an obstacle (Bosco et al., 2006) or it could be a copy of the motor signal that triggers flexion in the forelegs (Drew, 1993). We favor the latter possibility, because of the strong anatomical connections from the motor cortex and extensive evidence that efference copy is used to update target locations during memory guided eye movements (Colby and Goldberg, 1999; Crapse and Sommer, 2008) and emerging evidence of the use of motor output to predict the sensory consequences of hand or finger movements (Wolpert and Flanagan, 2001; Bays and Wolpert, 2006).

The conceptual model we propose is consistent with conclusions on the role of the PPC in guiding arm and eye movements in monkeys and humans. There is considerable evidence from primate studies that the PPC has a role in the maintenance of sensory representations of objects and body required for motor tasks. Two specific examples come from Wolpert et al. (1998) and Graziano et al. (2000). Using behavioral studies of a human with parietal lobe damage (Wolpert et al., 1998) and single-unit recordings from area 5 in monkeys (Graziano et al., 2000), both studies concluded the posterior parietal lobe in primates has a role in maintaining an internal representation of the body's state. Other studies have concluded that the PPC also maintains inter- 
nal representations of egocentric locations established visually (Stein, 1992; Buneo and Andersen, 2006; Burgess, 2006; Wolbers et al., 2008) and by previous movements (Rushworth et al., 1997).

\section{References}

Andujar JE, Drew T (2007) Organization of the projections from the posterior partietal cortex to the rostral and caudal regions of the motor cortex of the cat. J Comp Neurol 504:17-41.

Avendaño C, Rausell E, Perez-Aguilar D, Isorna S (1988) Organization of the association cortical afferent connection of area 5: a retrograde tracer study in the cat. J Comp Neurol 278:1-33.

Barash S, Bracewell RM, Fogassi L, Gnadt JW, Andersen RA (1991) Saccaderelated activity in the lateral intraparietal area. II. Spatial properties. J Neurophysiol 66:1109-1124.

Battaglia-Mayer A, Caminiti R (2002) Optic ataxia as a result of the breakdown of the global tuning fields of parietal neurons. Brain 125:225-237.

Bays PM, Wolpert DM (2006) Actions and consequences in bimanual interaction are represented in different coordinate systems. J Neurosci 26:7121-7126.

Beloozerova IN, Sirota MG (2003) Integration of motor and visual information in the parietal area 5 during locomotion. J Neurophysiol 90:961-971.

Bosco G, Eian J, Poppele RE (2006) Phase-specific sensory representations in spinocerebellar activity during stepping: evidence for a hybrid kinematic/kinetic model. Exp Brain Res 175:83-96.

Buneo CA, Andersen RA (2006) The posterior parietal cortex: sensorimotor interface for the planning and online control of visually guided movements. Neuropsychologia 44:2594-2606.

Burgess N (2006) Spatial memory: how egocentric and allocentric combine. Trends Cogn Sci 10:551-557.

Cinelli ME, Patla AE (2008) Locomotor avoidance behaviours during a visually guided task involving an approaching object. Gait Posture 28:596-601.

Colby CL, Goldberg ME (1999) Space and attention in parietal cortex. Annu Rev Neurosci 22:319-349.

Constantinidis C, Steinmetz MA (1996) Neuronal activity in posterior parietal area 7A during the delay periods of a spatial memory task. J Neurophysiol 76:1352-1355.

Crammond DJ, Kalaska JF (1989) Neuronal activity in primate parietal cortex area 5 varies with intended movement direction during an instructeddelay period. Exp Brain Res 76:458 - 462 .

Crapse TB, Sommer MA (2008) Corollary discharge across the animal kingdom. Nat Rev Neurosci 9:587-600.

Drew T (1993) Motor cortical activity during voluntary gait modifications in the cat. I. Cells related to the forelimbs. J Neurophysiol 70:179-199.

Drew T, Andujar JE, Lajoie K, Yakovenko S (2008) Cortical mechanisms involved in visuomotor coordination during precision walking. Brain Res Rev 57:199-211.

Fabre M, Buser P (1981) Effects of lesioning the anterior suprasylvian cortex on visuo-motor guidance performance in the cat. Exp Brain Res 41:81-88.

Fiset S, Doré FY (2006) Duration of cats (Felus catus) working memory for disappearing objects. Anim Cogn 9:62-70.

Fowler GA, Sherk H (2003) Gaze during visually-guided locomotion in cats. Behav Brain Res 139:83-96.

Ghosh S (1997) Identification of motor areas of the cat cerebral cortex based on studies of cortical stimulation and corticospinal connections. J Comp Neurol 380:191-214.

Gnadt JW, Andersen RA (1988) Memory related motor planning activity in posterior parietal cortex of macaque. Exp Brain Res 70:216-220.
Graziano MS, Cooke DF, Taylor CS (2000) Coding the location of the arm by sight. Science 290:1782-1786.

Kakei S, Shinoda Y (1990) Parietal projection of thalamocortical fibers from the ventroanterior-ventrolateral complex of the cat thalamus. Neurosci Lett 117:280-284.

Kalaska JF (1996) Parietal cotex area 5 and visuomotor behavior. Can J Physiol Pharmacol 74:483-498.

Kalaska JF, Crammond DJ (1995) Deciding not to go: neuronal correlates of response selection in a $\mathrm{GO} / \mathrm{NOGO}$ task in primate premotor and parietal cortex. Cereb Cortex 5:410-428.

Lajoie K, Drew T (2007) Lesions in area 5 of the posterior parietal cortex in the cat produce errors in the accuracy of paw placement during visuallyguided locomotion. J Neurophysiol 97:2339-2354.

Lajoie K, Andujar J, Pearson KG, Drew T (2007) Persistent neuronal activity in posterior parietal cortex area 5 related to long-lasting memories of obstacles in walking cats. Soc Neurosci Abstr 37:397.8.

Li CS, Mazzoni P, Andersen RA (1999) Effect of reversible inactivation of macaque lateral intraparietal area on visual and memory saccades. J Neurophysiol 81:1827-1838.

Marigold DS, Patla AE (2008) Visual information from the lower visual filed is important for walking across multi-sensory terrain. Exp Brain Res 188:23-31.

Marigold DS, Weerdesteyn V, Patla AE, Duysens J (2007) Keep looking ahead? Re-direction of visual fixation does not always occur during an unpredictable obstacle avoidance task. Exp Brain Res 176:32-42.

McVea DA, Pearson KG (2006) Long-lasting memories of obstacles guide leg movements in the walking cat. J Neurosci 26:1175-1178.

McVea DA, Pearson KG (2007) Stepping of the forelegs over obstacles establishes long-lasting memories in cats. Curr Biol 17:R621-R623.

Pasternak T, Greenlee MW (2005) Working memory in primate sensory systems. Nat Rev Neurosci 6:97-107.

Patla AE (1997) Understanding the roles of vision in the control of human locomotion. Gait Posture 5:54-69.

Patla AE, Greig M (2006) Any way you look at it, successful negotiation needs visually guided on-line foot placement regulation during the approach phase. Neurosci Lett 397:110-114.

Patla AE, Vickers JN (1997) Where and when do we look as we approach and step over an obstacle in the travel path? Neuroreport 8:3661-3665.

Pearson KG, McVea DA (2007) Posterior parietal cortical area 5 is necessary for long-lasting memories of obstacles in walking cats. Soc Neurosci Abstr 37:397.9.

Rushworth MF, Nixon PD, Passingham RE (1997) Parietal cortex and movement. I. Movement selection and reaching. Exp Brain Res 117:292-310.

Stein JF (1992) The representation of egocentric space in the posterior parietal cortex. Behav Brain Sci 15:691-700.

Wilkinson EJ, Sherk HA (2005) The use of visual information for planning accurate steps in a cluttered environment. Behav Brain Res 164:270-274.

Wolbers T, Hegarty M, Büchel C, Loomis JM (2008) Spatial undating: how the brain keeps track of changing object locations during observer motion. Nat Neurosci 11:1223-1230.

Wolpert DM, Flanagan JR (2001) Motor prediction. Curr Biol 11: R729-R732.

Wolpert DM, Goodbody SJ, Husain M (1998) Maintaining internal representations: the role of the human superior parietal lobe. Nat Neurosci $1: 529-533$.

Zhang M, Barash S (2004) Persistent LIP activity in memory antisaccades: working memory for sensorimotor transformations. J Neurophysiol 91: $1424-1441$. 